\title{
Prevalence and determinants of dental caries experience among adolescents in Kazakhstan: a cross-sectional study
}

\author{
Anara Zhumadilova ( $\square$ azhumadilova@nu.edu.kz ) \\ Nazarbayev University https://orcid.org/0000-0001-7514-9705 \\ Turgan Supiyev \\ Kazakh Medical University Continuing Education \\ Sholpan Abralina \\ Semey Medical University \\ Ardak Yeslyamgaliyeva \\ Astana medical university \\ Aizhan Kulmirzayeva \\ Astana medical university \\ Adil Supiyev \\ Nazarbayev University National Laboratory Astana
}

\section{Research article}

Keywords: DMF, Adolescents, caries prevalence, socioeconomic determinants, dental health, Central Asia, Kazakhstan.

Posted Date: May 10th, 2020

DOI: https://doi.org/10.21203/rs.3.rs-25373/v1

License: () This work is licensed under a Creative Commons Attribution 4.0 International License. Read Full License

Version of Record: A version of this preprint was published on February 15th, 2021. See the published version at https://doi.org/10.37290/ctnr2641-452X.19:388-397. 


\section{Abstract}

Background The information on dental caries experience and its determinants in Central Asia is limited. The aim of the present study was to provide estimates of the mean levels, prevalence and severity of dental caries, and to identify the factors associated with these characteristics in the population of school-aged children from four regions in Kazakhstan.

Methods We examined 2149 schoolchildren aged 11-15 years from 4 regions of Kazakhstan. The overall response rate was $68 \%$. Dental caries experience was assessed clinically, and standardized questionnaire was used to gather sociodemographic and oral health behavior information. Caries experience was measured by using DMFT (decayed, missing, and filled teeth) index. Negative binomial hurdle regression model was used to assess the relationship between the caries experience and covariates.

Results The overall prevalence of dental caries was $74 \%$, obvious decayed teeth was found in $44 \%$ of subjects, while only $6 \%$ had missing teeth and $24 \%$ had their teeth treated. The mean DMFT index was 2.48 among 12-year-olds and 3.9 among 15year-olds. There were large differences of dental caries experience between the regions, with all indicators (decayed, missed and filled teeth) being less favourable in Semey region. The caries prevalence experience in the studied population was associated with higher age, geographic region, ethnicity, mother's education, material deprivation, frequent consumption of sugar-added drinks, age of start brushing the teeth and dental attendance pattern. Age, region, ethnicity, dietary habits and dental attendance pattern were found to be significantly associated with the predicted degree of caries experience among those with caries experience.

Conclusions The high prevalence of dental caries and large differences in caries indices between the regions in Kazakhstan might be related to rapid westernization in the Central Asian region and associated nutrition transition. Also, the increasing burden of untreated caries in less advantaged populations was observed. Parent education and material deprivation may be a very important determinants of the oral health among adolescents in Kazakhstan.

\section{Background}

Caries is preventable condition, but still remains the most prevalent among the dental diseases $(1,2)$ resulting in expensive treatments and other negative consequences affecting the overall health (3). World Health Organization (WHO) stated that dental caries is pandemic mostly affecting schoolchildren with very high prevalence up to $90 \%(2,3)$. Dental caries is a multifactorial disease, various factors contributing to tooth decay development in children was found at individual level, including oral health behaviours and dietary habits, $(4,5)$ as well as numerous studies demonstrated strong association of dental caries experience with socioeconomic markers. $(6,7)$. Many of these risk factors might be related to societal changes after the collapse of the Soviet Union, when the newly independent states faced a rapid nutrition transition shifting towards westernized diets and increased consumption of sugar-added drinks and foods (8-10). The implications of these rapidly changing behaviours is not only the high prevalence, but also more severe dental caries experience $(5,11)$. According to the WHO oral health surveillance databank (12) Kazakhstan had one of the highest DMFT index among 12-year-olds equal to 4.0 (2015), while neighbouring Russia had 2.5 (2008) and China had 0.9 (2015); in turn the DMFT index in some developed countries was as low as 0.5 in UK and 0.7 in Sweden (2017). This routine information in other Central Asian countries was not available. Providing the evidence on the rates of caries experience and associated factors are very important, since modifying these risk factors may provide great potential to prevent the disease in Central Asian region and elsewhere (13-16).

Despite the high prevalence of dental caries and the importance of oral health for the quality of live, aesthetics and treatment cost issues, there are very few reliable individual-level data on this condition in the Central Asian countries $(6,17)$. The best available information on dental caries in this region were either outdated $(18,19)$, or the methodology of clinical examination and statistical analysis of the results was unclear (20). Importantly, given the positively skewed distribution of DMFT scoring and a large number of zero values the appropriate statistical analyses should be considered for correct interpretation and understanding of caries risk factors $(5,11,20)$. The aim of the present study was to provide estimates of the mean levels, 
prevalence and severity of dental caries, and to identify the factors associated with these characteristics in the population of school-aged children from four regions in Kazakhstan.

\section{Methods}

\section{Kazakhstan Adolescent Health Study}

The dataset used for this study is the Kazakhstan Adolescent Health Study (KAHS) implemented by the Laboratory of Epidemiology and Public health at the National Laboratory Astana in Nazarbayev University in collaboration with University College London, UK. The study was mainly based on the protocol of the Health Behaviour in School-Aged Children study (HBSC) (21), a WHO Collaborative Cross-National study, covering 44 countries and focusing on understanding adolescents' health in social context, the protocol was taken from the HBSC study after agreement with HBSC steering committee. For the dental health part of the KAHS study the Children's Dental Health Survey (CDHS) protocol was adapted and included both, the questionnaires for schoolchildren and their parents $(22,23)$.

\section{Study population}

The data for this cross-sectional survey were collected between 2014 and 2015. The target population was children between 11 and 15 years of age residing in the five regions of Kazakhstan: Nur-Sultan (capital city) and Kokshetau (North of Kazakhstan), Oskemen (East of Kazakhstan), Semey (East of Kazakhstan) and Qaragandy. Qaragandy was not included in the current analysis as it did not have the data on dental examination. Two schools of two different types were selected in each region. One type of school was Nazarbayev Intellectual School (NIS), a special type of school owned by the government, but with a distinct private structure, designed specifically for talented students. The second type of school was a regular public school, which serves the general population in each region. Both types of schools do not require any entry fees and are free of charge throughout the whole period of education.

Access to the parents of the adolescents in the target age group was achieved through parents-teacher meetings specially organized at each participating school. Parents were provided with oral and written information about the study prior being asked to consent for their child taking part in the study. Structured questionnaires contained sections on socio-demographic, socioeconomic, health, behavioural, and environmental information. Objective measurements were taken by trained staff and health professionals and included height, weight, waist and hip circumferences, blood pressure measurements, spirometry, swabs of buccal epithelium, hair samples and comprehensive dental health examination. In total, 2149 adolescents aged 1115 years participated in the study. The overall response rate was $68 \%$.

\section{Clinical Examination}

Clinical examination was carried out by 8 calibrated dentists in total, two in each participated region. Familiar adult, such as a school nurse or a teacher were present during the oral examination. All children were explained prior to examination about process of dental observation to reduce the anxiety. Each intra-oral clinical examination was performed with the child seated in a conventional school chair facing a window with sunlight access under standard conventional light with the dentist wearing a headlight. The examination was carried out using a sterilized, disposable set consisting of an illuminated mouth mirror and a blunt ball-ended probe with an end diameter of $0.5 \mathrm{~mm}$ (24). The assessments were performed only on cooperative basis to ensure the wellbeing of the children after parental approval. The dentist recorded the findings for each child on the scoring sheet.

\section{Dental caries definition}

In our study the dental caries experience was measured by the number of decayed (into dentine), missing, and filled teeth (DMFT), where the maximum score of DMFT could be up to 28 (wisdom teeth were excluded due to uneruption) and the minimum score equal zero. The diagnostic "into-dentine" threshold was based on caries scores at the D3 level based on WHO classification of dental caries $(4,25)$, i.e., non-cavitated dentine lesions and cavitated dentin lesions.

\section{Socio-demographic characteristics}


The socio-demographic and behaviour characteristics included age, sex, area of residence and several socioeconomic and demographic variables. The ethnicity of the child was self-reported and classified as Kazakh, Russian and other. Marital status of parents was classified as married or unmarried (widowed, divorced and single) and parental education was divided into school/secondary or less, vocational and university. Material deprivation was used as markers of the economic status and was self-reported in the parental questionnaire. Material deprivation was assessed by three questions (how often participants do not have enough money for food, clothes and paying bills for their households), and participants were classified into three categories (high, intermediate and low).

\section{Dietary habits and oral health behaviour}

Sugar added foods included questions on the consumption of cakes, biscuits, chocolate, and sweets, and sugary drinks included questions on the consumption fruit juices and carbonated soft drinks. Based on the frequency the sugar-added drinks consumption was grouped into three categories: "less than 1 time a day", "once to three times per day", and "four times or more per day". Similarly, the frequency of sugar-added foods consumption was categorized as "less than 1 time a day", "once to three times per day", and "four times or more per day". Frequency of tooth-brushing was classified as "three or more" "twice a day", and "one or less per day" while age when first started to brush the teeth was categorized as "under 2 years", 2-4 years", and "over 4 years". Pattern of dental visits was defined as "regular", "occasional" or "never".

\section{Ethics}

The study protocol was approved by the ethical committee of the Centre for Life Sciences, Nazarbayev University. Permissions and approvals were also obtained from school administrative staff. Prior to participation, parents were provided with detailed information about the study in Kazakh and Russian languages. Written consent was obtained for each participant.

\section{Statistical analyses}

In the descriptive analyses, unadjusted frequencies of all covariates (separately for socio-demographic and behaviour factors) were stratified by DMF categories, mean caries experience, percentages of caries-free (DMFT $=0)$ participants were also reported. The Mann-Whitney U and Kruskal-Wallis tests were used to assess statistical significance, depending on the frequency distribution, the chi-square test was used for categorical variables.

The significant likelihood-ratio test of alpha (chi-square $=195.34, p<0.0001$ ) implied that the analysed data was over-dispersed and the use of negative binomial regression over Poisson regression was most accurate. A negative binomial hurdle model $(\mathrm{NBH})$ was used to investigate the effect of selected variables on dental caries experience, which has recently been suggested as an accurate approach that has a better fit to these type of count data, accommodate many zero counts and has fewer misleading interpretations. Hurdle models have the advantage of estimating two separate parameters; one-part (logistic regression) models only zeros, and the second part (zero-truncated negative binomial regression) models the values over zero. The logistic regression model was generated for caries-free cases, predicting whether or not the child was in the "caries-free" group; as all zero values were modeled separately from the caries counts, the inferences for the caries-free prevalence was done directly for our study population. The association between outcome (caries-free individuals) and covariates (sociodemographic characteristics, dietary habits and oral health behaviours) were adjusted for age and sex, region and school type. (Table 3, column 2) An additional logistic regression model adjusting for all covariates was also estimated. (Table 3, column 3) The second part was generated to predict the greater caries experience for adolescents with dental caries experience. (Table 3 , column 4) To adjust the standard errors so that they are robust to heteroscedasticity as well as autocorrelation within clusters the robust variance estimator was used. All analyses were performed using STATA software, version 14 (College Station, Texas, USA). 
Table 3

Association between the DMFT index and socio-demographic and behavioural factors in the negative binomial hurdle models

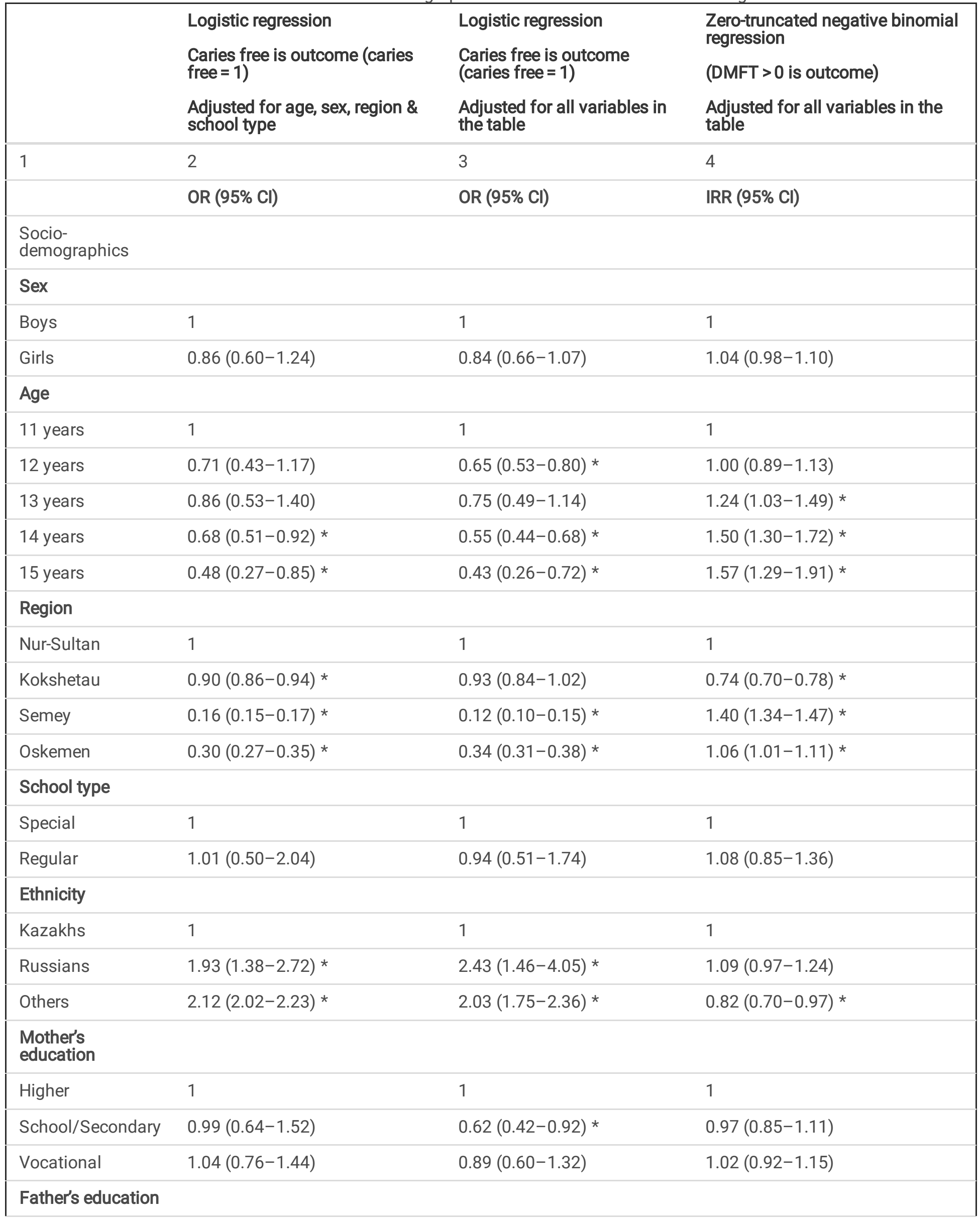




\begin{tabular}{|c|c|c|c|}
\hline & $\begin{array}{l}\text { Logistic regression } \\
\text { Caries free is outcome (caries } \\
\text { free }=1 \text { ) }\end{array}$ & $\begin{array}{l}\text { Logistic regression } \\
\text { Caries free is outcome } \\
\text { (caries free }=1 \text { ) }\end{array}$ & $\begin{array}{l}\text { Zero-truncated negative binomial } \\
\text { regression } \\
\text { (DMFT >0 is outcome) }\end{array}$ \\
\hline & $\begin{array}{l}\text { Adjusted for age, sex, region \& } \\
\text { school type }\end{array}$ & $\begin{array}{l}\text { Adjusted for all variables in } \\
\text { the table }\end{array}$ & $\begin{array}{l}\text { Adjusted for all variables in the } \\
\text { table }\end{array}$ \\
\hline Higher & 1 & 1 & 1 \\
\hline School/Secondary & $1.14(0.76-1.72)$ & $1.31(0.88-1.95)$ & $0.94(0.86-1.03)$ \\
\hline Vocational & $1.23(1.11-1.35)$ * & $1.13(0.95-1.33)$ & $0.90(0.83-0.98)$ \\
\hline \multicolumn{4}{|l|}{$\begin{array}{l}\text { Parent marital } \\
\text { status }\end{array}$} \\
\hline Married & 1 & 1 & 1 \\
\hline Unmarried & $1.27(1.09-1.49)$ * & $1.48(0.82-2.68)$ & $0.95(0.75-1.20)$ \\
\hline \multicolumn{4}{|l|}{ Deprivation level } \\
\hline Highly deprived & 1 & 1 & 1 \\
\hline Intermediate & $1.40(1.15-1.70)$ * & $1.42(1.05-1.90)$ * & $0.98(0.92-1.06)$ \\
\hline Less deprived & $1.10(0.92-1.33)$ & $1.17(1.06-1.29)$ * & $1.00(0.98-1.02)$ \\
\hline \multicolumn{4}{|l|}{$\begin{array}{l}\text { Behavioural } \\
\text { factors }\end{array}$} \\
\hline \multicolumn{4}{|l|}{ Daily sugar drinks } \\
\hline$<1$ time a day & 1 & 1 & 1 \\
\hline $1-3$ times a day & $0.82(0.62-1.09)$ & $0.80(0.50-1.28)$ & $1.04(0.96-1.13)$ \\
\hline > 3 times a day & $0.64(0.51-0.80)$ * & $0.55(0.36-0.86)$ * & $0.91(0.84-1.00)$ * \\
\hline \multicolumn{4}{|l|}{ Daily sugar foods } \\
\hline$<1$ time a day & 1 & 1 & 1 \\
\hline $1-3$ times a day & $0.96(0.84-1.10)$ & $1.03(0.80-1.31)$ & $0.97(0.95-0.99)$ * \\
\hline$>3$ times a day & $1.00(0.75-1.33)$ & $0.99(0.71-1.37)$ & $1.14(1.04-1.25)$ * \\
\hline \multicolumn{4}{|l|}{ Age start brushing } \\
\hline Under 2 years & 1 & 1 & 1 \\
\hline $2-4$ years & $0.68(0.49-0.97)$ * & $0.71(0.46-1.09)$ & $1.06(0.96-1.18)$ \\
\hline Over 4 years & $0.66(0.42-1.03)$ & $0.67(0.41-1.09)$ & $1.03(0.97-1.09)$ \\
\hline \multicolumn{4}{|l|}{$\begin{array}{l}\text { Brushing } \\
\text { frequency }\end{array}$} \\
\hline Three or more & 1 & 1 & 1 \\
\hline Two times & $0.92(0.50-1.70)$ & $0.92(0.47-1.80)$ & $0.97(0.91-1.03)$ \\
\hline One or less & $0.99(0.63-1.54)$ & $0.97(0.57-1.67)$ & $0.88(0.73-1.07)$ \\
\hline \multicolumn{4}{|l|}{ Dentist visits } \\
\hline Regular & 1 & 1 & 1 \\
\hline Occasional & $1.29(0.95-1.75)$ * & $1.46(1.08-1.98)$ * & $0.96(0.88-1.05)$ * \\
\hline
\end{tabular}




\begin{tabular}{|c|c|c|c|}
\hline & $\begin{array}{l}\text { Logistic regression } \\
\text { Caries free is outcome (caries } \\
\text { free }=1 \text { ) }\end{array}$ & $\begin{array}{l}\text { Logistic regression } \\
\text { Caries free is outcome } \\
\text { (caries free }=1 \text { ) }\end{array}$ & $\begin{array}{l}\text { Zero-truncated negative binomial } \\
\text { regression } \\
\text { (DMFT > } 0 \text { is outcome) }\end{array}$ \\
\hline & $\begin{array}{l}\text { Adjusted for age, sex, region \& } \\
\text { school type }\end{array}$ & $\begin{array}{l}\text { Adjusted for all variables in } \\
\text { the table }\end{array}$ & $\begin{array}{l}\text { Adjusted for all variables in the } \\
\text { table }\end{array}$ \\
\hline Never & $2.93(1.46-5.89)$ * & $3.80(1.90-7.59)$ * & $0.74(0.63-0.87)$ * \\
\hline
\end{tabular}

\section{Results}

Table 1 shows the DMFT status and mean DMFT scores classified by socio-demographic characteristics of the school-based samples of adolescents' population in the four distinct regions in Kazakhstan. The age of the participants was between 1115 years. A total of 2149 children were included in the analysis, the majority were females $(n=1186)$, from Nur-sultan region ( $n$ $=721)$, of Kazakh ethnicity $(n=1798)$ and in special schools $(n=1325)$. The parents' higher education level was the most frequently reported category, more common among mother's $(n=1431)$ compared to fathers $(n=1173)$. Very few parents $(n=$ 315) reported their marital status to be unmarried and based on selected socioeconomic marker only small number of participants were classified to be in highly deprived group $(n=296)$. 
Table 1

Socio-demographic characteristics associated with dental caries experience measured by DMFT index in Kazakhstan

\begin{tabular}{|c|c|c|c|c|c|c|c|c|c|c|}
\hline & $+N(n)$ & $\begin{array}{l}\text { DMFT } \\
\text { mean } \\
(\mathrm{SD})\end{array}$ & $\begin{array}{l}\text { *p- } \\
\text { value }\end{array}$ & $\begin{array}{l}\text { DMFT } \\
>0 \\
\text { mean } \\
(\mathrm{SD})\end{array}$ & $\begin{array}{l}\text { *p- } \\
\text { value }\end{array}$ & $\begin{array}{l}\text { Caries } \\
\text { free } \\
\%(\mathrm{Cl})\end{array}$ & $\begin{array}{l}\text { **p- } \\
\text { value }\end{array}$ & $\begin{array}{l}\text { Decayed } \\
\%(\mathrm{Cl})\end{array}$ & $\begin{array}{l}\text { Missing } \\
\%(\mathrm{Cl})\end{array}$ & $\begin{array}{l}\text { Filled } \\
\%(\mathrm{Cl})\end{array}$ \\
\hline \multirow[t]{2}{*}{ Overall } & 2149 & 3.09 & & 4.20 & & 26.4 & & 44.0 & 6.0 & 23.6 \\
\hline & $(1,582)$ & $(3.06)$ & & $(2.84)$ & & $\begin{array}{l}(24.6- \\
28.3)\end{array}$ & & $\begin{array}{l}(41.9- \\
46.1)\end{array}$ & $\begin{array}{l}(5.1- \\
7.1)\end{array}$ & $\begin{array}{l}(21.9- \\
25.5)\end{array}$ \\
\hline Sex & & & 0.0765 & & 0.4443 & & 0.096 & & & \\
\hline \multirow[t]{2}{*}{ Boys } & 963 & 2.97 & & 4.13 & & 28.1 & & 44.3 & 5.4 & 22.1 \\
\hline & (692) & $(3.00)$ & & $(2.79)$ & & $\begin{array}{l}(25.4- \\
31.1)\end{array}$ & & $\begin{array}{l}(41.2- \\
47.5)\end{array}$ & $\begin{array}{l}(4.1- \\
7.0)\end{array}$ & $\begin{array}{l}(19.6- \\
24.9)\end{array}$ \\
\hline \multirow[t]{2}{*}{ Girls } & 1186 & 3.19 & & 4.26 & & 25.0 & & 43.7 & 6.5 & 24.9 \\
\hline & (890) & $(3.11)$ & & $(2.89)$ & & $\begin{array}{l}(22.6- \\
27.5)\end{array}$ & & $\begin{array}{l}(40.9- \\
46.5)\end{array}$ & $\begin{array}{l}(5.2- \\
8.0)\end{array}$ & $\begin{array}{l}(22.5- \\
27.4)\end{array}$ \\
\hline Age & & & 0.0001 & & 0.0001 & & 0.103 & & & \\
\hline \multirow[t]{2}{*}{11 years } & 189 & 2.36 & & 3.46 & & 31.7 & & 45.0 & 3.7 & 19.6 \\
\hline & (129) & $(2.28)$ & & $(1.94)$ & & $\begin{array}{l}(25.5- \\
38.7)\end{array}$ & & $\begin{array}{l}(38.0- \\
52.1)\end{array}$ & $\begin{array}{l}(1.8- \\
7.6)\end{array}$ & $\begin{array}{l}(14.5- \\
25.9)\end{array}$ \\
\hline \multirow[t]{2}{*}{12 years } & 542 & 2.48 & & 3.36 & & 26.2 & & 43.2 & 4.1 & 26.6 \\
\hline & $(400)$ & $(2.29)$ & & $(2.04)$ & & $\begin{array}{l}(22.7- \\
30.1)\end{array}$ & & $\begin{array}{l}(39.1- \\
47.4)\end{array}$ & $\begin{array}{l}(2.7- \\
6.1)\end{array}$ & $\begin{array}{l}(23.0- \\
30.5)\end{array}$ \\
\hline \multirow[t]{2}{*}{13 years } & 532 & 2.94 & & 4.12 & & 28.6 & & 43.2 & 6.2 & 22.0 \\
\hline & $(380)$ & $(2.91)$ & & $(2.64)$ & & $\begin{array}{l}(24.9- \\
32.6)\end{array}$ & & $\begin{array}{l}(39.1- \\
47.5)\end{array}$ & $\begin{array}{l}(4.4- \\
8.6)\end{array}$ & $\begin{array}{l}(18.7- \\
25.7)\end{array}$ \\
\hline \multirow[t]{2}{*}{14 years } & 555 & 3.60 & & 4.82 & & 25.2 & & 45.4 & 6.5 & 22.9 \\
\hline & (415) & $(3.46)$ & & (3.19) & & $\begin{array}{l}(21.8- \\
29.0)\end{array}$ & & $\begin{array}{l}(41.3- \\
49.6)\end{array}$ & $\begin{array}{l}(4.7- \\
8.9)\end{array}$ & $\begin{array}{l}(19.6- \\
26.6)\end{array}$ \\
\hline \multirow[t]{2}{*}{15 years } & 331 & 3.90 & & 5.01 & & 22.1 & & 43.5 & 9.4 & 25.1 \\
\hline & (258) & $(3.70)$ & & $(3.47)$ & & $\begin{array}{l}(17.9- \\
26.9)\end{array}$ & & $\begin{array}{l}(38.2- \\
48.9)\end{array}$ & $\begin{array}{l}(6.7- \\
13.0)\end{array}$ & $\begin{array}{l}(20.7- \\
30.0)\end{array}$ \\
\hline Region & & & 0.0001 & & 0.0001 & & 0.000 & & & \\
\hline \multirow[t]{2}{*}{ Nur-Sultan } & 721 & 2.45 & & 3.98 & & 38.3 & & 30.1 & 2.8 & 28.8 \\
\hline & $(445)$ & $(3.02)$ & & $(2.95)$ & & $\begin{array}{l}(34.8- \\
41.9)\end{array}$ & & $\begin{array}{l}(26.9- \\
33.6)\end{array}$ & $\begin{array}{l}(1.8- \\
4.3)\end{array}$ & $\begin{array}{l}(25.7- \\
32.3)\end{array}$ \\
\hline
\end{tabular}

Notes: DMFT - into-dentin Decayed, Missing, and Filled permanent teeth,

$+\mathrm{N}$ is the total number of participants; and in brackets is the number of participants with DMFT >0;

* $\mathrm{p}$-value were obtained using Mann-Whitney-Wilcoxon test for two groups; and Kruskal-Wallis $\mathrm{H}$ test for more than two groups;

** p-value were obtained using Pearson's chi-square test (proportion of caries free individuals; i.e. DMFT = 0); 


\begin{tabular}{|c|c|c|c|c|c|c|c|c|c|c|}
\hline & $\mathrm{tN}(\mathrm{n})$ & $\begin{array}{l}\text { DMFT } \\
\text { mean } \\
(S D)\end{array}$ & $\begin{array}{l}\text { *p- } \\
\text { value }\end{array}$ & $\begin{array}{l}\text { DMFT } \\
>0 \\
\text { mean } \\
(S D)\end{array}$ & $\begin{array}{l}\text { *p- } \\
\text { value }\end{array}$ & $\begin{array}{l}\text { Caries } \\
\text { free } \\
\%(\mathrm{Cl})\end{array}$ & $\begin{array}{l}\text { **p- } \\
\text { value }\end{array}$ & $\begin{array}{l}\text { Decayed } \\
\%(\mathrm{Cl})\end{array}$ & $\begin{array}{l}\text { Missing } \\
\%(\mathrm{Cl})\end{array}$ & $\begin{array}{l}\text { Filled } \\
\%(\mathrm{Cl})\end{array}$ \\
\hline \multirow[t]{2}{*}{ Kokshetau } & 463 & 1.88 & & 2.96 & & 36.5 & & 36.5 & 7.1 & 19.9 \\
\hline & (294) & $(2.11)$ & & $(1.96)$ & & $\begin{array}{l}(32.2- \\
41.0)\end{array}$ & & $\begin{array}{l}(32.2- \\
41.0)\end{array}$ & $\begin{array}{l}(5.1- \\
9.9)\end{array}$ & $\begin{array}{l}(16.5- \\
23.8)\end{array}$ \\
\hline \multirow[t]{2}{*}{ Semey } & 551 & 4.80 & & 5.30 & & 9.4 & & 69.9 & 10.2 & 10.5 \\
\hline & $(499)$ & (3.23) & & $(2.98)$ & & $\begin{array}{l}(7.3- \\
12.2)\end{array}$ & & $\begin{array}{l}(65.9- \\
73.6)\end{array}$ & $\begin{array}{l}(7.9- \\
13.0)\end{array}$ & $\begin{array}{l}(8.2- \\
13.4)\end{array}$ \\
\hline \multirow[t]{2}{*}{ Oskemen } & 414 & 3.29 & & 3.96 & & 16.9 & & 42.0 & 4.8 & 36.2 \\
\hline & (344) & $(2.78)$ & & $(2.58)$ & & $\begin{array}{l}(13.6- \\
20.8)\end{array}$ & & $\begin{array}{l}(37.4- \\
46.9)\end{array}$ & $\begin{array}{l}(3.1- \\
7.4)\end{array}$ & $\begin{array}{l}(31.7- \\
41.0)\end{array}$ \\
\hline School type & & & 0.7965 & & 0.5159 & & 0.387 & & & \\
\hline \multirow[t]{2}{*}{ Special } & 1325 & 3.07 & & 4.13 & & 25.7 & & 42.6 & 5.1 & 26.6 \\
\hline & (984) & $(2.98)$ & & $(2.75)$ & & $\begin{array}{l}(23.5- \\
28.2)\end{array}$ & & $\begin{array}{l}(39.9- \\
45.2)\end{array}$ & $\begin{array}{l}(4.0- \\
6.4)\end{array}$ & $\begin{array}{l}(24.3- \\
29.1)\end{array}$ \\
\hline \multirow[t]{2}{*}{ Regular } & 824 & 3.13 & & 4.32 & & 27.4 & & 46.2 & 7.5 & 18.8 \\
\hline & (598) & (3.19) & & $(2.98)$ & & $\begin{array}{l}(24.5- \\
30.6)\end{array}$ & & $\begin{array}{l}(42.9- \\
49.7)\end{array}$ & $\begin{array}{l}(5.9- \\
9.5)\end{array}$ & $\begin{array}{l}(16.3- \\
21.6)\end{array}$ \\
\hline Ethnicity & & & 0.0001 & & 0.1592 & & $\begin{array}{l}< \\
0.0001\end{array}$ & & & \\
\hline \multirow[t]{2}{*}{ Kazakh } & 1798 & 3.21 & & 4.23 & & 24.2 & & 44.5 & 6.3 & 25.0 \\
\hline & $(1,363)$ & (3.08) & & $(2.86)$ & & $\begin{array}{l}(22.2- \\
26.2)\end{array}$ & & $\begin{array}{l}(42.3- \\
46.9)\end{array}$ & $\begin{array}{l}(5.3- \\
7.5)\end{array}$ & $\begin{array}{l}(23.0- \\
27.0)\end{array}$ \\
\hline \multirow[t]{2}{*}{ Russian } & 237 & 2.70 & & 4.16 & & 35.0 & & 45.1 & 4.6 & 15.2 \\
\hline & (154) & (3.04) & & $(2.85)$ & & $\begin{array}{l}(29.2- \\
41.3)\end{array}$ & & $\begin{array}{l}(38.9- \\
51.5)\end{array}$ & $\begin{array}{l}(2.6- \\
8.2)\end{array}$ & $\begin{array}{l}(11.2- \\
20.4)\end{array}$ \\
\hline \multirow[t]{2}{*}{ Others } & 114 & 2.04 & & 3.58 & & 43.0 & & 32.5 & 4.4 & 20.2 \\
\hline & (65) & $(2.55)$ & & $(2.42)$ & & $\begin{array}{l}(34.2- \\
52.3)\end{array}$ & & $\begin{array}{l}(24.5- \\
41.6)\end{array}$ & $\begin{array}{l}(1.8- \\
10.1)\end{array}$ & $\begin{array}{l}(13.8- \\
28.6)\end{array}$ \\
\hline $\begin{array}{l}\text { Mother's } \\
\text { education }\end{array}$ & & & 0.0014 & & 0.0414 & & 0.030 & & & \\
\hline \multirow[t]{2}{*}{ Higher } & 1431 & 3.03 & & 4.09 & & 25.9 & & 41.7 & 5.0 & 27.5 \\
\hline & $(1,061)$ & $(2.99)$ & & $(2.78)$ & & $\begin{array}{l}(23.7- \\
28.2)\end{array}$ & & $\begin{array}{l}(39.2- \\
44.3)\end{array}$ & $\begin{array}{l}(3.9- \\
6.2)\end{array}$ & $\begin{array}{l}(25.2- \\
29.8)\end{array}$ \\
\hline
\end{tabular}

Notes: DMFT - into-dentin Decayed, Missing, and Filled permanent teeth,

$+\mathrm{N}$ is the total number of participants; and in brackets is the number of participants with DMFT >0;

* $\mathrm{p}$-value were obtained using Mann-Whitney-Wilcoxon test for two groups; and Kruskal-Wallis $\mathrm{H}$ test for more than two groups;

** p-value were obtained using Pearson's chi-square test (proportion of caries free individuals; i.e. DMFT = 0); 


\begin{tabular}{|c|c|c|c|c|c|c|c|c|c|c|}
\hline & $+N(n)$ & $\begin{array}{l}\text { DMFT } \\
\text { mean } \\
(\mathrm{SD})\end{array}$ & $\begin{array}{l}\text { *p- } \\
\text { value }\end{array}$ & $\begin{array}{l}\text { DMFT } \\
>0 \\
\text { mean } \\
(S D)\end{array}$ & $\begin{array}{l}\text { *p- } \\
\text { value }\end{array}$ & $\begin{array}{l}\text { Caries } \\
\text { free } \\
\%(C l)\end{array}$ & $\begin{array}{l}\text { **p- } \\
\text { value }\end{array}$ & $\begin{array}{l}\text { Decayed } \\
\%(\mathrm{Cl})\end{array}$ & $\begin{array}{l}\text { Missing } \\
\%(\mathrm{Cl})\end{array}$ & $\begin{array}{l}\text { Filled } \\
\%(\mathrm{Cl})\end{array}$ \\
\hline \multirow[t]{2}{*}{ School/Secondary } & 150 & 3.41 & & 4.30 & & 20.7 & & 54.7 & 8.0 & 16.7 \\
\hline & (119) & $(3.03)$ & & $(2.78)$ & & $\begin{array}{l}(14.9- \\
27.9)\end{array}$ & & $\begin{array}{l}(46.6- \\
62.5)\end{array}$ & $\begin{array}{l}(4.6- \\
13.6)\end{array}$ & $\begin{array}{l}(11.5- \\
23.5)\end{array}$ \\
\hline \multirow[t]{2}{*}{ Vocational } & 418 & 3.67 & & 4.60 & & 20.1 & & 52.6 & 10.0 & 17.2 \\
\hline & (334) & $(3.29)$ & & $(3.05)$ & & $\begin{array}{l}(16.5- \\
24.2)\end{array}$ & & $\begin{array}{l}(47.8- \\
57.4)\end{array}$ & $\begin{array}{l}(7.5- \\
13.3)\end{array}$ & $\begin{array}{l}(13.9- \\
21.2)\end{array}$ \\
\hline Father's education & & & 0.0179 & & 0.1519 & & 0.085 & & & \\
\hline \multirow[t]{2}{*}{ Higher } & 1173 & 3.16 & & 4.21 & & 24.8 & & 41.3 & 5.3 & 28.6 \\
\hline & (882) & $(3.08)$ & & $(2.87)$ & & $\begin{array}{l}(22.4- \\
27.4)\end{array}$ & & $\begin{array}{l}(38.5- \\
44.1)\end{array}$ & $\begin{array}{l}(4.1- \\
6.7)\end{array}$ & $\begin{array}{l}(26.1- \\
31.3)\end{array}$ \\
\hline \multirow[t]{2}{*}{ School/Secondary } & 181 & 3.73 & & 4.54 & & 17.7 & & 64.6 & 7.2 & 10.5 \\
\hline & (149) & $(2.99)$ & & $(2.68)$ & & $\begin{array}{l}(12.8- \\
24.0)\end{array}$ & & $\begin{array}{l}(57.4- \\
71.3)\end{array}$ & $\begin{array}{l}(4.2- \\
12.0)\end{array}$ & $\begin{array}{l}(6.8- \\
15.9)\end{array}$ \\
\hline \multirow[t]{2}{*}{ Vocational } & 419 & 3.26 & & 4.19 & & 22.2 & & 51.3 & 7.9 & 18.6 \\
\hline & $(326)$ & $(3.05)$ & & $(2.84)$ & & $\begin{array}{l}(18.5- \\
26.4)\end{array}$ & & $\begin{array}{l}(46.5- \\
56.1)\end{array}$ & $\begin{array}{l}(5.6- \\
10.9)\end{array}$ & $\begin{array}{l}(15.2- \\
22.6)\end{array}$ \\
\hline $\begin{array}{l}\text { Parent Marital } \\
\text { status }\end{array}$ & & & 0.0010 & & 0.0314 & & 0.011 & & & \\
\hline \multirow[t]{2}{*}{ Married } & 1,695 & 3.27 & & 4.25 & & 23.2 & & 46.1 & 6.1 & 24.6 \\
\hline & $(1302)$ & $(3.07)$ & & $(2.84)$ & & $\begin{array}{l}(21.2- \\
25.3)\end{array}$ & & $\begin{array}{l}(43.8- \\
48.5)\end{array}$ & $\begin{array}{l}(5.0- \\
7.3)\end{array}$ & $\begin{array}{l}(22.6- \\
26.7)\end{array}$ \\
\hline \multirow[t]{2}{*}{ Unmarried } & 315 & 2.76 & & 3.93 & & 29.8 & & 39.7 & 7.0 & 23.5 \\
\hline & $(221)$ & $(3.03)$ & & $(2.90)$ & & $\begin{array}{l}(25.0- \\
35.1)\end{array}$ & & $\begin{array}{l}(34.4- \\
45.2)\end{array}$ & $\begin{array}{l}(4.6- \\
10.4)\end{array}$ & $\begin{array}{l}(19.1- \\
28.5)\end{array}$ \\
\hline Deprivation level & & & 0.7157 & & 0.5037 & & 0.159 & & & \\
\hline \multirow[t]{2}{*}{ Highly deprived } & 296 & 3.36 & & 4.23 & & 20.6 & & 54.1 & 6.8 & 18.6 \\
\hline & (235) & $(3.33)$ & & $(3.20)$ & & $\begin{array}{l}(16.4- \\
25.6)\end{array}$ & & $\begin{array}{l}(48.3- \\
59.7)\end{array}$ & $\begin{array}{l}(4.4- \\
10.2)\end{array}$ & $\begin{array}{l}(14.5- \\
23.4)\end{array}$ \\
\hline \multirow[t]{2}{*}{ Intermediate } & 669 & 3.17 & & 4.30 & & 26.3 & & 42.6 & 7.9 & 23.2 \\
\hline & (493) & $(3.09)$ & & $(2.84)$ & & $\begin{array}{l}(23.1- \\
29.8)\end{array}$ & & $\begin{array}{l}(38.9- \\
46.4)\end{array}$ & $\begin{array}{l}(6.1- \\
10.2)\end{array}$ & $\begin{array}{l}(20.1- \\
26.5)\end{array}$ \\
\hline
\end{tabular}

Notes: DMFT - into-dentin Decayed, Missing, and Filled permanent teeth,

$+\mathrm{N}$ is the total number of participants; and in brackets is the number of participants with DMFT >0;

* $\mathrm{p}$-value were obtained using Mann-Whitney-Wilcoxon test for two groups; and Kruskal-Wallis $\mathrm{H}$ test for more than two groups;

** p-value were obtained using Pearson's chi-square test (proportion of caries free individuals; i.e. DMFT = 0); 


\begin{tabular}{|c|c|c|c|c|c|c|c|c|c|c|}
\hline & $+\mathrm{N}(\mathrm{n})$ & $\begin{array}{l}\text { DMFT } \\
\text { mean } \\
(S D)\end{array}$ & $\begin{array}{l}\text { *p- } \\
\text { value }\end{array}$ & $\begin{array}{l}\text { DMFT } \\
>0 \\
\text { mean } \\
(\mathrm{SD})\end{array}$ & $\begin{array}{l}\text { *p- } \\
\text { value }\end{array}$ & $\begin{array}{l}\text { Caries } \\
\text { free } \\
\%(C l)\end{array}$ & $\begin{array}{l}\text { **p- } \\
\text { value }\end{array}$ & $\begin{array}{l}\text { Decayed } \\
\%(\mathrm{Cl})\end{array}$ & $\begin{array}{l}\text { Missing } \\
\%(\mathrm{Cl})\end{array}$ & $\begin{array}{l}\text { Filled } \\
\%(\mathrm{Cl})\end{array}$ \\
\hline \multirow[t]{2}{*}{ Less deprived } & 1024 & 3.14 & & 4.14 & & 24.1 & & 44.2 & 5.1 & 26.6 \\
\hline & $(777)$ & $(2.98)$ & & $(2.75)$ & & $\begin{array}{l}(21.6- \\
26.8)\end{array}$ & & $\begin{array}{l}(41.2- \\
47.3)\end{array}$ & $\begin{array}{l}(3.9- \\
6.6)\end{array}$ & $\begin{array}{l}(23.9- \\
29.4)\end{array}$ \\
\hline \multicolumn{11}{|c|}{ Notes: DMFT - into-dentin Decayed, Missing, and Filled permanent teeth, } \\
\hline \multicolumn{11}{|c|}{$+\mathrm{N}$ is the total number of participants; and in brackets is the number of participants with DMFT >0; } \\
\hline \multicolumn{11}{|c|}{$\begin{array}{l}\text { * p-value were obtained using Mann-Whitney-Wilcoxon test for two groups; and Kruskal-Wallis } \mathrm{H} \text { test for more than two } \\
\text { groups; }\end{array}$} \\
\hline \multicolumn{11}{|c|}{ ** p-value were obtained using Pearson's chi-square test (proportion of caries free individuals; i.e. DMFT = 0); } \\
\hline
\end{tabular}

Dental caries experience was measured using DMFT status and mean DMFT index in the analysis. Additionally, the mean DMFT scores in adolescents with DMFT $>0$ were calculated. Caries-free subjects represented only $26 \%$, almost half (44\%) of the examined subjects had obvious decayed teeth experience, while $6 \%$ had missing and $24 \%-$ filled teeth. $26 \%$ of caries-free adolescents among 12-year-olds vs. $22 \%$ among 15 -year-olds were observed in the study, the mean DMFT score for 12- and 15year-olds was 2.5 and 3.9 respectively. While the number of the obvious decay experience and treated teeth (filled) were very similar between 12 and 15 years-olds, there were twice the difference in the proportions of missing teeth between these two age groups, $4 \%$ among 12-year-olds and $9 \%$ among 15-year-olds. The distribution of DMFT status across regions demonstrated that Nur-Sultan (38\%) and Kokshetau (37\% ) regions had considerably higher proportion of caries-free children, however the treatment levels (filled teeth) were the highest in Oskemen region; besides the lowest mean DMFT score was in Kokshetau region (1.9) and contrarily the highest score was in Semey region (4.8). Despite small differences of obvious decay experience between regular (46\%) and special (43\%) schools, there were large gap in treatment levels, $19 \%$ in regular vs. $27 \%$ in special schools. There were also marked differences in DMFT status between adolescents' ethnical groups: in spite of higher proportion of caries free participants among Russians (35\%) compared to Kazakhs (24\%), there were opposite results of treatment levels between the groups, $15 \%$ in Russians vs. $25 \%$ in Kazakhs. Consistently for both parents the higher education level was slightly advantageous, with more caries free children and much higher treatment levels.

Table 2 represents the DMFT status and mean DMFT index by oral health behaviour factors. Children's caries experience varied by behaviour factors; those who reported frequent added sugar diets (over 3 times a day) such as daily sugar drinks and daily sugar foods, had less caries-free adolescents (21\% and $24 \%$ respectively) and higher DMFT scores (3.1 and 3.6 respectively) compared to those who consumed sugar diets less frequently (less than 1 time a day). Adolescents who reported regular dental visits had lower prevalence of caries free teeth (23\%) and higher DMFT index (3.3) as well as more filled teeth (35\%) compared to those who reported occasional or no visits at all. Age when start brushing teeth were more consistent marker of oral health compared to daily brushing frequency, both DMFT index and DMFT status showed large gap between early and late start of toothbrushing, 2.8 vs 3.4 DMFT score and $31 \%$ vs $20 \%$ of caries-free subjects respectively. 
Table 2

Lifestyle behaviour factors associated with dental caries experience measured by DMFT index in Kazakhstan

\begin{tabular}{|c|c|c|c|c|c|c|c|c|c|c|}
\hline & $+N(n)$ & $\begin{array}{l}\text { DMFT } \\
\text { mean } \\
(S D)\end{array}$ & $\begin{array}{l}{ }^{* p} \text { - } \\
\text { value }\end{array}$ & $\begin{array}{l}\text { DMFT } \\
>0 \\
\text { mean } \\
(S D)\end{array}$ & $\begin{array}{l}\text { *p- } \\
\text { value }\end{array}$ & $\begin{array}{l}\begin{array}{l}\text { Caries } \\
\text { free }\end{array} \\
\% \text { (Cl) }\end{array}$ & $\begin{array}{l}\text { **p- } \\
\text { value }\end{array}$ & $\begin{array}{l}\text { Decayed } \\
\%(\mathrm{Cl})\end{array}$ & $\begin{array}{l}\text { Missing } \\
\%(\mathrm{Cl})\end{array}$ & $\begin{array}{l}\text { Filled } \\
\%(\mathrm{Cl})\end{array}$ \\
\hline $\begin{array}{l}\text { Daily sugar } \\
\text { drinks }\end{array}$ & & & 0.1605 & & 0.8116 & & 0.072 & & & \\
\hline \multirow[t]{2}{*}{$<1$ time a day } & 755 & 2.93 & & 4.11 & & 28.9 & & 42.4 & 4.2 & 24.5 \\
\hline & $(537)$ & $(2.96)$ & & $(2.72)$ & & $\begin{array}{l}(25.7- \\
32.2)\end{array}$ & & $\begin{array}{l}(38.9- \\
45.9)\end{array}$ & $\begin{array}{l}(3.0- \\
5.9)\end{array}$ & $\begin{array}{l}(21.6- \\
27.7)\end{array}$ \\
\hline \multirow{2}{*}{$\begin{array}{l}1-3 \text { times a } \\
\text { day }\end{array}$} & 1232 & 3.19 & & 4.28 & & 25.6 & & 43.8 & 6.7 & 23.9 \\
\hline & (917) & $(3.18)$ & & $(2.98)$ & & $\begin{array}{l}(23.2- \\
28.1)\end{array}$ & & $\begin{array}{l}(41.1- \\
46.6)\end{array}$ & $\begin{array}{l}(5.5- \\
8.3)\end{array}$ & $\begin{array}{l}(21.6- \\
26.3)\end{array}$ \\
\hline \multirow[t]{2}{*}{$>3$ times a day } & 162 & 3.13 & & 3.96 & & 21.0 & & 52.5 & 8.6 & 17.9 \\
\hline & $(128)$ & $(2.62)$ & & $(2.32)$ & & $\begin{array}{l}(15.4- \\
28.0)\end{array}$ & & $\begin{array}{l}(44.8- \\
60.1)\end{array}$ & $\begin{array}{l}(5.2- \\
14.1)\end{array}$ & $\begin{array}{l}(12.7- \\
24.6)\end{array}$ \\
\hline $\begin{array}{l}\text { Daily sugar } \\
\text { foods }\end{array}$ & & & 0.0243 & & 0.0337 & & 0.209 & & & \\
\hline \multirow[t]{2}{*}{$<1$ time a day } & 885 & 2.95 & & 4.11 & & 28.2 & & 39.9 & 5.0 & 26.9 \\
\hline & $(635)$ & $(2.97)$ & & $(2.75)$ & & $\begin{array}{l}(25.4- \\
31.3)\end{array}$ & & $\begin{array}{l}(36.7- \\
43.2)\end{array}$ & $\begin{array}{l}(3.7- \\
6.6)\end{array}$ & $\begin{array}{l}(24.1- \\
29.9)\end{array}$ \\
\hline \multirow{2}{*}{$\begin{array}{l}1-3 \text { times a } \\
\text { day }\end{array}$} & 943 & 3.05 & & 4.10 & & 25.6 & & 45.5 & 6.3 & 22.7 \\
\hline & $(702)$ & $(2.99)$ & & $(2.78)$ & & $\begin{array}{l}(22.9- \\
28.4)\end{array}$ & & $\begin{array}{l}(42.3- \\
48.7)\end{array}$ & $\begin{array}{l}(4.9- \\
8.0)\end{array}$ & $\begin{array}{l}(20.1- \\
25.5)\end{array}$ \\
\hline \multirow[t]{2}{*}{$>3$ times a day } & 321 & 3.60 & & 4.71 & & 23.7 & & 50.8 & 8.1 & 17.4 \\
\hline & $(245)$ & $(3.44)$ & & $(3.20)$ & & $\begin{array}{l}(19.3- \\
28.6)\end{array}$ & & $\begin{array}{l}(45.3- \\
56.2)\end{array}$ & $\begin{array}{l}(5.6- \\
11.6)\end{array}$ & $\begin{array}{l}(13.7- \\
22.0)\end{array}$ \\
\hline $\begin{array}{l}\text { Age start } \\
\text { brushing }\end{array}$ & & & 0.0001 & & 0.1227 & & $\begin{array}{l}< \\
0.0001\end{array}$ & & & \\
\hline \multirow[t]{2}{*}{ Under 2 years } & 703 & 2.80 & & 4.03 & & 30.6 & & 38.7 & 4.7 & 26.0 \\
\hline & $(488)$ & $(3.00)$ & & $(2.82)$ & & $\begin{array}{l}(27.3- \\
34.1)\end{array}$ & & $\begin{array}{l}(35.2- \\
42.4)\end{array}$ & $\begin{array}{l}(3.4- \\
6.5)\end{array}$ & $\begin{array}{l}(22.9 \\
29.4)\end{array}$ \\
\hline \multirow[t]{2}{*}{$2-4$ years } & 876 & 3.34 & & 4.26 & & 21.5 & & 47.1 & 6.5 & 24.9 \\
\hline & (688) & $(3.11)$ & & $(2.91)$ & & $\begin{array}{l}(18.8- \\
24.3)\end{array}$ & & $\begin{array}{l}(43.9- \\
50.5)\end{array}$ & $\begin{array}{l}(5.1- \\
8.3)\end{array}$ & $\begin{array}{l}(22.1- \\
27.9)\end{array}$ \\
\hline \multirow[t]{2}{*}{ Over 4 years } & 429 & 3.44 & & 4.29 & & 19.8 & & 51.3 & 8.2 & 20.7 \\
\hline & $(344)$ & $(3.00)$ & & $(2.76)$ & & $\begin{array}{l}(16.3- \\
23.9)\end{array}$ & & $\begin{array}{l}(46.5- \\
56.0)\end{array}$ & $\begin{array}{l}(5.9- \\
11.2)\end{array}$ & $\begin{array}{l}(17.2- \\
24.9)\end{array}$ \\
\hline \multicolumn{11}{|c|}{ Notes: DMFT - into-dentin Decayed, Missing, and Filled permanent teeth, } \\
\hline \multicolumn{11}{|c|}{$+\mathrm{N}$ is the total number of participants; and in brackets is the number of participants with DMFT >0; } \\
\hline \multicolumn{11}{|c|}{$\begin{array}{l}\text { * p-value were obtained using Mann-Whitney-Wilcoxon test for two groups; and Kruskal-Wallis } \mathrm{H} \text { test for more than two } \\
\text { groups; }\end{array}$} \\
\hline
\end{tabular}




\begin{tabular}{|c|c|c|c|c|c|c|c|c|c|c|}
\hline & $+N(n)$ & $\begin{array}{l}\text { DMFT } \\
\text { mean } \\
(S D)\end{array}$ & $\begin{array}{l}{ }^{*} \mathrm{p}- \\
\text { value }\end{array}$ & $\begin{array}{l}\text { DMFT } \\
>0 \\
\text { mean } \\
(\mathrm{SD})\end{array}$ & $\begin{array}{l}{ }^{* p} \text { - } \\
\text { value }\end{array}$ & $\begin{array}{l}\begin{array}{l}\text { Caries } \\
\text { free }\end{array} \\
\%(\mathrm{Cl})\end{array}$ & $\begin{array}{l}\text { **p- } \\
\text { value }\end{array}$ & $\begin{array}{l}\text { Decayed } \\
\%(\mathrm{Cl})\end{array}$ & $\begin{array}{l}\text { Missing } \\
\%(\mathrm{Cl})\end{array}$ & $\begin{array}{l}\text { Filled } \\
\%(\mathrm{Cl})\end{array}$ \\
\hline $\begin{array}{l}\text { Daily brushing } \\
\text { frequency }\end{array}$ & & & 0.0242 & & 0.0206 & & 0.367 & & & \\
\hline \multirow[t]{2}{*}{ Three or more } & 269 & 3.41 & & 4.59 & & 25.7 & & 44.2 & 5.6 & 24.5 \\
\hline & $(200)$ & $(3.43)$ & & $(3.22)$ & & $\begin{array}{l}(20.8- \\
31.2)\end{array}$ & & $\begin{array}{l}(38.4- \\
50.2)\end{array}$ & $\begin{array}{l}(3.4- \\
9.1)\end{array}$ & $\begin{array}{l}(19.8- \\
30.0)\end{array}$ \\
\hline \multirow[t]{2}{*}{ Two times } & \multirow{2}{*}{$\begin{array}{l}1,218 \\
(908)\end{array}$} & 3.19 & & 4.28 & & 25.5 & & 43.5 & 5.7 & 25.3 \\
\hline & & $(3.12)$ & & $(2.90)$ & & $\begin{array}{l}(23.1- \\
28.0)\end{array}$ & & $\begin{array}{l}(40.8- \\
46.3)\end{array}$ & $\begin{array}{l}(4.6- \\
7.2)\end{array}$ & $\begin{array}{l}(22.9- \\
27.8)\end{array}$ \\
\hline \multirow[t]{2}{*}{ One or less } & 662 & 2.79 & & 3.89 & & 28.4 & & 44.7 & 6.6 & 20.2 \\
\hline & $(474)$ & $(2.76)$ & & $(2.52)$ & & $\begin{array}{l}(25.1- \\
32.0)\end{array}$ & & $\begin{array}{l}(41.0- \\
48.5)\end{array}$ & $\begin{array}{l}(5.0- \\
8.8)\end{array}$ & $\begin{array}{l}\left(17.3^{-}\right. \\
23.5)\end{array}$ \\
\hline Dentist visits & & & 0.0095 & & 0.4167 & & 0.008 & & & \\
\hline \multirow[t]{2}{*}{ Regular } & 492 & 3.34 & & 4.32 & & 22.8 & & 37.6 & 4.3 & 35.4 \\
\hline & $(380)$ & $(3.13)$ & & $(2.90)$ & & $\begin{array}{l}(19.3- \\
26.7)\end{array}$ & & $\begin{array}{l}(33.4- \\
42.0)\end{array}$ & $\begin{array}{l}(2.8- \\
6.5)\end{array}$ & $\begin{array}{l}(31.3- \\
39.7)\end{array}$ \\
\hline \multirow[t]{2}{*}{ Occasional } & 1552 & 3.05 & & 4.17 & & 26.8 & & 45.5 & 6.6 & 21.1 \\
\hline & $(1136)$ & $(3.05)$ & & $(2.83)$ & & $\begin{array}{l}(24.7- \\
29.1)\end{array}$ & & $\begin{array}{l}(43.0- \\
48.0)\end{array}$ & $\begin{array}{l}(5.5- \\
8.0)\end{array}$ & $\begin{array}{l}(19.1- \\
23.2)\end{array}$ \\
\hline \multirow[t]{2}{*}{ Never } & 105 & 2.52 & & 4.02 & & 37.1 & & 51.4 & 4.8 & 6.7 \\
\hline & $(66)$ & $(2.88)$ & & $(2.68)$ & & $\begin{array}{l}(28.4- \\
46.8)\end{array}$ & & $\begin{array}{l}(41.9- \\
60.9)\end{array}$ & $\begin{array}{l}(2.0- \\
11.0)\end{array}$ & $\begin{array}{l}(3.2- \\
.13 .4)\end{array}$ \\
\hline \multicolumn{11}{|c|}{ Notes: DMFT - into-dentin Decayed, Missing, and Filled permanent teeth, } \\
\hline \multicolumn{11}{|c|}{$+\mathrm{N}$ is the total number of participants; and in brackets is the number of participants with DMFT >0; } \\
\hline \multicolumn{11}{|c|}{$\begin{array}{l}\text { * p-value were obtained using Mann-Whitney-Wilcoxon test for two groups; and Kruskal-Wallis } \mathrm{H} \text { test for more than two } \\
\text { groups; }\end{array}$} \\
\hline
\end{tabular}

Table 3 summarizes the results of adjusted odds ratio (OR) and incidence rate ratio (IRR) obtained using the negative binomial hurdle model and presented separately for the logistic and zero-truncated negative binomial parts. The logistic regression part was represented by two models, first model adjusted for age, sex, region and school type, second model was adjusted for all socio-demographic factors including selected socioeconomic markers and major behavioural factors pertinent for oral health.

In adjusted model, the prevalence of caries was associated with higher age, geographic region, ethnicity, material deprivation frequent consumption of sugar-added drinks, age of start brushing the teeth and dental attendance pattern. Multivariable analysis of caries prevalence (Table 3, column 3) largely confirmed these findings, except that the association with ethnicity, material deprivation, mother's education level and frequent consumption of sugar-added drinks were increased, while age of start brushing the teeth was attenuated.

Further, in the second part of the NBH model several factors were found to be significantly associated with the predicted degree of caries experience among those with caries experience. The predicted degree of caries experience was gradually increased by age, up to 1.57 (95\% $\mathrm{Cl}: 1.29-1.91)$ times higher for those at the age of 15 years old compared to the youngest age group, 
however the difference was not significant between 11 and 12 years-olds. The variations between the regions were consistent with the first logit part of the model and represented the large differences between the regions, with marginally higher adjusted DMFT index in Oskemen region, 1.06 (95\% Cl: 1.01-1.11) and the highest score in Semey region, 1.41 (95\% Cl: 1.29-1.91) times higher than in Nur-Sultan region, it also confirmed the lower adjusted DMFT index in Kokshetau region compared to neighbouring capital region, 0.73 (95\% Cl: 0.70-0.77). Despite, no significant effect was found for Russians, adolescents of other than Kazakh and Russian ethnical groups had an adjusted DMFT index that was 0.82 (95\% Cl: 0.69-0.97) times lower than that observed among Kazakhs.

Again, the significant effects were found for some behaviour risk factors. While adolescents who reported most frequent daily sugar drinks consumption had slightly lower 0.91 (95\% Cl: 0.83-1.00) times lower the predicted degree of caries experience compared to those with less frequent levels; frequent daily sugar foods also had significant effect on DMFT index, those who reported frequent daily sugar had 1.14 (95\% Cl: 1.04-1.24) times higher DMFT index compared to reference group.

Furthermore, adolescents who reported occasional or no dental visits had an adjusted DMFT index that was 0.96 (95\% Cl: $0.88-1.05)$ and $0.74(95 \% \mathrm{Cl}: 0.63-0.86)$ times lower than that observed in those who reported regular visits.

\section{Discussion}

In this school-based cross-sectional study of adolescents' population from the four regions in Kazakhstan, we found the overall prevalence of dental caries of $74 \%$ out of which $44 \%$ of subjects had obvious decayed teeth experience, while only $6 \%$ had missing teeth and $24 \%$ had their teeth treated (filled). The mean DMFT index was 2.48 among 12-year-olds and 3.9 among 15year-olds. There were large differences of dental caries experience between the regions, with all indicators (decayed, missed and filled teeth) being less favourable in Semey region. Most associations of having no caries with covariates were in expected direction, the caries prevalence experience in our population was associated with higher age, geographic region, ethnicity, material deprivation frequent consumption of sugar-added drinks, age of start brushing the teeth and dental attendance pattern. Age, region, ethnicity, dietary habits and dental attendance pattern were found to be significantly associated with the predicted degree of caries experience among those with caries experience.

The prevalence of dental caries in our study was higher than in many developed $(4,22,23)$ and some developing countries (12), but our results were very similar to other post-soviet countries. The prevalence of caries among 12-year-olds in Kazakhstan (73.8\%) was roughly analogous to Moldova (77.5\%), Russia (77.5\%) and Georgia (68.9\%) [(26-28)], and slightly lower compared to Lithuania (85.5\%), Belarus (85\%) and Latvia (91.2\%) [(29-31)]. The 15-year-olds from Kazakhstan had higher prevalence of caries (77.9\%) compared to another Central Asian country Uzbekistan (68\%) (32), but lower than Moldova (86.2\%), Russia (91.8\%), Georgia (82.3\%) and Armenia (90.8\%) $(26-28,33)$. These similarities might be explained by the common challenges in transition period after the dissolution of the Soviet Union, when the access to public dental services for children dropped dramatically and dental healthcare became mostly private. Concurrently, the rapid increase in the availability of sugar-sweetened beverages and refined carbohydrate foods largely contributed to dental caries experience $(34,35)$.

The results of our study should be interpreted in view of several limitations. Firstly, the cross-sectional study design may not be appropriate way to estimate the causal relationships, yet the age of starting to brush the teeth or parent socio-demographic factors is likely to precede the development of the outcome, however we are less confident about the remaining covariates. Secondly, the response rate in this study was moderate (68\%), however many recent studies in both developed and developing countries reported roughly the same percentage $(8,9,23,36)$. The moderate response rates may lead to both under- and overestimation of caries prevalence, but usually non-responders in dental health studies have poorer oral health than responders (37). The response rates were slightly higher in special schools, but the difference was not sufficient to introduce the selection bias. Third, adjustment for potential confounders may be incomplete; for example, the sources of fluoride were not identified in this study, which could have modified the results (38), urban-rural differences would also be another interesting aspect to consider in the future research.

The inclusion of two cities in each geographic area in this study was made intentionally to represent more affluent and larger urban centres as well as smaller peripheral cities, for example Nur-Sultan (the capital city) and Kokshetau in the North of

Page 14/19 
Kazakhstan, and Oskemen (regional administrative centre) and Semey in the East of Kazakhstan region. The odds of observing no caries experience was significantly lower for adolescents who resided in the East-Kazakhstan region, with the lowest odds in

Semey city. Besides, residing in Semey city contributed significantly to the amount of caries; in the count part of NBH model the adjusted rate ratio was 1.41, while the difference between the capital city and regional administrative centre Oskemen was rather modest, 1.06 . The lower levels of caries experience and caries severity in particular in the capital city and neighbouring Kokshetau could be attributed to a wealthier population in this region compared to East-Kazakhstan, with better access to dental health services and more sophisticated system of private dental health care, the similar trend was observed in other developing countries (39). Further, the level of treatment (filled teeth) in both Nur-Sultan and Oskemen region were very high compared to their counterparts Kokshetau and Semey, while the proportion of missing teeth in these smaller peripheral settings exceeds that in large urban centres more than twice. The reduced public provision of dental services in less developed settings might increase the treatment needs (34), also the lack of oral health awareness among parents and poor health seeking behaviours might be another cause $(34,40)$.

Our findings on association of caries experience and its degree with oral health behaviours were consistent with previous research $(36,41,42)$. Many recent studies showed strong association of dental caries experience with a frequent consumption of sugar-added foods and drinks, the behaviours that usually acquired in early childhood $(43,44)$. In our study frequent consumption of sugar-added drinks were strongly associated with lower prevalence of carries-free individuals, though it had opposite effect on caries severity in zero-truncated negative binomial model. On the other hand, frequent consumption of sugaradded foods showed its strong effect on caries severity, while no association was found with caries prevalence.

Although the tooth brushing is considered to be highly effective $(5,36,42)$ and widely available and affordable method associated with prevention of dental caries (45), it wasn't associated with both caries experience and caries severity in our study. By contrast, the association of dental caries experience with age when children were accustomed to brush the teeth was significant and benefited those who adapted the habit earlier in life, this was reported previously in the literature $(5,42)$. Interestingly, the regular dental attendance pattern was strongly associated with the higher DMFT which undoubtedly may suggest that dental services are focused on treatment in the studied region rather than prevention, as stated in some studies $(11,39)$. Besides, the rare dental visits and lower treatment level were mostly observed in unmarried parents, parents with lower education level and highly deprived group with limited access to private dental care especially in less advantaged regions.

Disparities in adolescent's health according to socio-economic status are often reported $(14,15,23)$. Our study results of the effect of material deprivation on oral health inequalities was observed only in individuals who were disease free. The difference in prevalence of caries free individuals for each level of material deprivation wasn't incremental, with smaller difference between highly deprived and less deprived groups. This may suggest that there are some similarities in adverse oral health behaviours between these two groups $(14,34,39)$, however, as already mentioned, the level of treatment was much lower in highly deprived group. The caries experience was also varying among study ethnic groups with almost three-fold increase of caries-free individuals among Russians compared to Kazakhs in the fully-adjusted model, which may reflect the differences in lifestyle and particularly in nutrition, since the traditional Kazakh diet includes high consumption of energy-dense foods; on the contrary the caries severity was marginally (9\%) higher in Russians compared to Kazakhs, in addition, the treatment component of DMFT was also much lower among ethnic Russians.

\section{Conclusion}

The high prevalence of dental caries and large differences in caries indices between the regions in Kazakhstan might be related to rapid westernization in the Central Asian region and associated nutrition transition. Also, the increasing burden of untreated caries in less advantaged populations was observed. Parent education and material deprivation may be a very important determinants of the oral health among adolescents in Kazakhstan.

\section{Abbreviations}

CDHS

Page 15/19 
FDI

World Dental Association,

WHO

World Health Organization

KAHS

Kazakhstan Adolescent Health Study

DMFT

Decay, Missed, Filled teeth

\section{Declarations}

\section{Ethics approval and consent to participate}

The study protocol was approved by the ethical committee of the Centre for Life Sciences, Nazarbayev University. Permissions and approvals were also obtained from school administrative staff. Prior to participation, parents were provided with detailed information about the study in Kazakh and Russian languages. Written informed consent was obtained from a parent or guardian for participants under 16 years old.

\section{Consent for publication}

Not applicable

\section{Availability of data and material}

The datasets used and analysed during the current study are available from the corresponding author on reasonable request.

\section{Competing interests}

The authors declare that they have no competing interests.

\section{Funding}

This work was supported the research grant titled "Study of biomarkers associated with oral cavity diseases among the adolescents from different region of Kazakhstan" of the Ministry of Science and Education of the Republic of Kazakhstan (\# AP05132694). The funding body had no role in the design of the study, data collection, analysis and interpretation of results, nor in the writing of the manuscript. 


\section{Authors' contributions}

AZ and AS jointly designed the study, analysed the data, drafted and finalized the manuscript. AY and SA helped to conduct field work, recruiting of participants and clinical dental examination and critically revised the manuscript. TS and AK contributed to data analysis and critically revised the manuscript. All authors have read and approved the final manuscript.

\section{Acknowledgements}

The authors are grateful for administrative and medical staff of schools in Nur-Sultan, Kokshetau, Semey and Oskemen regions of Kazakhstan that participated in Kazakhstan Adolescent Health Study for their important contributions.

\section{References}

1. Anil S, Anand PS. Early Childhood Caries: Prevalence, Risk Factors, and Prevention. Front Pediatr. 2017;5:157.

2. Do LG, Ha DH, Spencer AJ. Factors attributable for the prevalence of dental caries in Queensland children. Community Dent Oral Epidemiol. 2015;43(5):397-405.

3. Gathecha G, Makokha A, Wanzala P, Omolo J, Smith P. Dental caries and oral health practices among 12 year old children in Nairobi West and Mathira West Districts, Kenya. Pan Afr Med J. 2012;12:42.

4. Elamin A, Garemo M, Gardner A. Dental caries and their association with socioeconomic characteristics, oral hygiene practices and eating habits among preschool children in Abu Dhabi, United Arab Emirates - the NOPLAS project. BMC Oral Health. 2018;18(1):104.

5. Hong J, Whelton H, Douglas G, Kang J. Consumption frequency of added sugars and UK children's dental caries. Community Dent Oral Epidemiol. 2018;46(5):457-64.

6. Petersson GH, Bratthall D. The caries decline: a review of reviews. Eur J Oral Sci. 1996;104(4 (Pt 2)):436-43.

7. Cleaton-Jones P, Fatti P. Dental caries trends in Africa. Community Dent Oral Epidemiol. 1999;27(5):316-20.

8. Supiyev A, Kossumov A, Kassenova A, Nurgozhin T, Zhumadilov Z, Peasey A, et al. Diabetes prevalence, awareness and treatment and their correlates in older persons in urban and rural population in the Astana region, Kazakhstan. Diabetes Res Clin Pract. 2016;112:6-12.

9. Supiyev A, Nurgozhin T, Zhumadilov Z, Peasey A, Hubacek JA, Bobak M. Prevalence, awareness, treatment and control of dyslipidemia in older persons in urban and rural population in the Astana region, Kazakhstan. BMC Public Health. 2017;17(1):651.

10. Popkin BM. Relationship between shifts in food system dynamics and acceleration of the global nutrition transition. Nutr Rev. 2017;75(2):73-82.

11. Drachev SN, Brenn T, Trovik TA. Dental caries experience and determinants in young adults of the Northern State Medical University, Arkhangelsk, North-West Russia: a cross-sectional study. BMC Oral Health. 2017;17(1):136.

12. Kassebaum NJ, Bernabé E, Dahiya M, Bhandari B, Murray CJ, Marcenes W. Global burden of untreated caries: a systematic review and metaregression. J Dent Res. 2015;94(5):650-8.

13. Moynihan P, Petersen PE. Diet, nutrition and the prevention of dental diseases. Public Health Nutr. 2004;7(1A):201-26.

14. Petersen PE. Sociobehavioural risk factors in dental caries - international perspectives. Community Dent Oral Epidemiol. 2005;33(4):274-9.

15. Petersen PE, Bourgeois D, Ogawa H, Estupinan-Day S, Ndiaye C. The global burden of oral diseases and risks to oral health. Bull World Health Organ. 2005;83(9):661-9.

16. Hobdell M, Petersen PE, Clarkson J, Johnson N. Global goals for oral health 2020. Int Dent J. 2003;53(5):285-8. 
17. Zharmagambetova A, Tuleutayeva S, Akhmetova S, Sumanova A, Baigulakov A, Sakenov T, et al. Prevalence and experience of dental caries among 12- and 15-year-old adolescents in Central Kazakhstan. Public Health. 2017;151:11820.

18. VA. K. Effect of micro elements in the drinking water of Dzhambul on the incidence of dental caries and fluorosis. Gig Sanit. ; 1972 p. 108-9.

19. AA K, KR A. Effects of air pollution on the etiology of dental caries. Gig Sanit. ; 1991.

20. Beaglehole RB, Crail H, Mackay J, Judith. The Oral Health Atlas. Mapping a neglected global health issue FDI World Dental Federation \& Myriad Editions 2009; 2009.

21. Roberts C, Freeman J, Samdal O, Schnohr CW, de Looze ME, Nic Gabhainn S, et al. The Health Behaviour in School-aged Children (HBSC) study: methodological developments and current tensions. Int J Public Health. 2009;54(Suppl 2):140-50.

22. Pitts N, Chadwick B, Anderson T. Children's Dental Health Survey 2013 Report 2: Dental Disease and Damage in Children England, Wales and Northern Ireland.. 2015.

23. Vernazza CR, Rolland SL, Chadwick B, Pitts N. Caries experience, the caries burden and associated factors in children in England, Wales and Northern Ireland 2013. Br Dent J. 2016;221(6):315-20.

24. Organization WH. Oral health survey: basic methods. 5th ed. Geneva: World Health Organization; 2013.

25. Chestnutt IG. Dental Public Health at a Glance: Wiley Blackwell, UK; 2016.

26. Bilder L, Stepco E, Uncuta D, Aizenbud D, Machtei E, Bilder A, et al. The pathfinder study among schoolchildren in the Republic of Moldova: dental caries experience. Int Dent J. 2018;68(5):344-7.

27. Gorbatova MA, Gorbatova LN, Grjibovski AM. Dental caries experience among 15-year-old adolescents in north-west Russia. Int J Circumpolar Health. 2011;70(3):232-5.

28. Sgan-Cohen HD, Margvelashvili V, Bilder L, Kalandadze M, Margvelashvili GM. M, et al. Dental caries among children in Georgia by age, gender, residence location and ethnic group. Community Dent Health. ; 2014. p. 163-6.

29. Milciuviene S, Bendoraitiene E, Andruskeviciene V, Narbutaite J, Sakalauskiene J, Vasiliauskiene I, et al. Dental caries prevalence among 12-15-year-olds in Lithuania between 1983 and 2005. Medicina. 2009;45(1):68-76.

30. Bondarik E, Leous P. Oral Health and Children Attitudes Among Mothers and Schoolteachers in Belarus. Stomatologija, Baltic Dent Maxillo J; 2004.

31. Berzina S, Care R. Dental Health in 11 and 13 Year Old Children in Latvia. Stomatologija 2003. p. 62-4.

32. Shadiev K, Leous P, Pakhomov G. Significance of the WHO criteria in national achievement of oral health objectives: experiences from Belarus and Uzbekistan / World Health Organization.. WHO Oral Health Programme \& WHO Collaborating Centre on Oral Health. https://apps.who.int/iris/handle/10665/644331998.

33. Manrikian ME, Markarian MM, Vardanian IF. The state of periodontal and tooth tissues after the introduction of preventive measures among the children of Tavoush region, Republic of Armenia. Georgian Med News. 2012(204):33-6.

34. Emerich K, Adamowicz-Klepalska B. Dental caries among 7-year-old children in northern Poland, 1987-2003. Public Health Rep. 2007;122(4):552-8.

35. Dragheim E, Petersen PE, Kalo I, Saag M. Dental caries in schoolchildren of an Estonian and a Danish municipality. Int J Paediatr Dent. 2000;10(4):271-7.

36. Li Y, Wulaerhan J, Liu Y, Abudureyimu A, Zhao J. Prevalence of severe early childhood caries and associated socioeconomic and behavioral factors in Xinjiang, China: a cross-sectional study. BMC Oral Health. 2017;17(1):144.

37. Splieth $\mathrm{CH}$, Steffen H, Welk A, Schwahn C. Responder and nonresponder analysis for a caries prevention program. Caries Res. 2005;39(4):269-72.

38. Petersen PE, Hiroshi O. Prevention of dental caries through the use of fluoride - the WHO approach. Community Dental Health 2016. p. 66-8.

39. Xavier Aea. Impact of dental caries on quality of life of adolescents according to access to oral health services: a cross sectional study. Braz. J. Oral Sci. 2016. 
40. Begzati A, Meqa K, Siegenthaler D, Berisha M, Mautsch W. Dental health evaluation of children in kosovo. Eur J Dent. 2011;5(1):32-9.

41. Shao RHT, Zhong YS, Li X, Gao YB, Wang YF, Yin W. Socio-demographic factors, dental status and health-related behaviors associated with geriatric oral health-related quality of life in Southwestern China. Health Qual Life Outcomes.2018.

42. Wulaerhan J, Abudureyimu A, Bao XL, Zhao J. Risk determinants associated with early childhood caries in Uygur children: a preschool-based cross-sectional study. BMC Oral Health. 2014;14:136.

43. Colak H, Dülgergil CT, Dalli M, Hamidi MM. Early childhood caries update: A review of causes, diagnoses, and treatments. J Nat Sci Biol Med. 2013;4(1):29-38.

44. Zukanović A. Caries risk assessment models in caries prediction. Acta Med Acad. 2013;42(2):198-208.

45. Polk DE, Geng M, Levy S, Koerber A, Flay BR. Frequency of daily tooth brushing: predictors of change in 9- to 11-year old US children. Community Dent Health. 2014;31(3):136-40. 